\title{
Queso fresco elaborado con sustitución parcial de aceite de Cucúrbita ficifolia B. "Calabaza" y su evaluación fisicoquímica y sensorial
}

\section{Fresh cheese made with partial substitution of Cucurbit ficifolia B. "Pumpkin" and its physicochemical and sensorial evaluation}

Luis Artica M. ${ }^{11}$

(1) Facultad de Ingeniería en Industrias Alimentarias Universidad Nacional del Centro del Perú.

Email: lartica@uncp.edu.pe

\section{RESUMEN}

El presente trabajo, tiene por objetivo elaborar queso fresco sustituyendo parcialmente con aceite de semilla de Cucúrbita ficifolia B. "Calabaza” y su evaluación fisicoquímica y sensorial. Para la elaboración del queso fresco prensado, se utilizó leche estandarizada al 3\% de grasa y leche descremada al $1 \%$; a los cuales, se incorporó el aceite de semilla de calabaza en niveles de $0,1 \% ; 0,5 \%$ y $0,7 \%$, obteniéndose seis tratamientos. Los resultados indican que la incorporación de aceite de semilla de calabaza no tiene influencia en la acidez del queso siendo un valor de $0,12 \%$ (expresado en ácido láctico). La composición química presenta diferencia estadística significativamente $(P<0.01)$ por efecto de la variación del porcentaje de grasa de la materia prima leche y porcentaje de incorporación del aceite de semilla de calabaza. Las proteínas varían de $17,34 \%$ a $421,12 \%$; la grasa de $6,30 \%$ a $14,30 \%$; lactosa de $6,67 \%$ a $7,37 \%$ y humedad de $55,33 \%$ a $65,62 \%$. Estos valores se encuentra dentro de los valores normativos vigentes, según la evaluación sensorial; para los atributos: color, olor, sabor y textura, se observa que hay diferencia significativa entre los tratamientos y el queso fresco elaborado con $1 \%$ de grasa y con $0,5 \%$ de incorporación de aceite de semilla de calabaza, presentando mayor aceptabilidad. Se concluye que el queso fresco elaborado con incorporación de aceite de semilla de calabaza presenta características fisicoquímicas y sensoriales aceptables, mejorando el valor nutritivo en relación a la calidad de la grasa.

Palabras Claves: Queso fresco - Aceite de semilla de calabaza - Evaluación fisicoquímica - Evaluación sensorial - Leche estandarizada y descremada.

\begin{abstract}
The present work aims to make fresh cheese by partially replacing it with Cucurbita ficifolia B. seed oil. "Calabaza" and its physicochemical and sensory evaluation. For the production of fresh cheese pressed in the research, standardized milk at 3\% fat and 1\% skimmed milk was used, to which the pumpkin seed oil was incorporated at levels of $0.1 \% ; 0.5 \%$ and $0.7 \%$, obtaining six treatments. The results indicate that the incorporation of pumpkin seed oil has no influence on the acidity of the cheese being a value of $0.12 \%$ (expressed in lactic acid). The chemical composition shows a statistically significant difference $(\mathrm{P}<0.01)$ due to the variation of the fat percentage of the milk raw material and percentage of incorporation of the pumpkin seed oil, the proteins vary from $17,34 \%$ to $421,12 \%$; the fat from $6.30 \%$ to $14.30 \%$; lactose from $6.67 \%$ to $7.37 \%$; Humidity from $55.33 \%$ to $65.62 \%$, these values are within the normative values in force. According to the sensory evaluation; For the attributes: color, smell, taste and texture, it is observed that there is a significant difference between the treatments and the fresh cheese made with $1 \%$ fat and with $0.5 \%$ incorporation of pumpkin seed oil, has greater acceptability. It is concluded that fresh cheese made with the incorporation of pumpkin seed oil presents acceptable physicochemical and sensory characteristics, improving the nutritional value in relation to the quality of the fat.
\end{abstract}

Keywords: Fresh cheese - Pumpkin seed oil - Physicochemical evaluation - sensory evaluation - Standardized and skim milk. 


\section{INTRODUCCIÓN}

En las últimas décadas, se han incrementado mucho interés por el estudio de los efectos de la grasa sobre la aparición de enfermedades cardiovasculares, los cuales se atribuyen a los alimentos con contenido de ácidos grasos saturados. El estudio se centró en definir la acción sobre lo lípidos plasmáticos, y se estableció la relación entre el colesterol y el riesgo cardiovascular; con lo que se demuestra que, las grasas son los nutrientes con mayor impacto sobre el metabolismo lipídico (Carrillo et al. 2010). Muchas publicaciones han demostrado que la relación entre salud e ingesta de grasa no depende tanto de su cantidad, sino de su calidad; es decir, del tipo de ácido graso predominante en la dieta (Pérez-Jiménez F, Ruano J, Pérez-Martínez P, López-Segura F, López-Miranda J; 2007). En ellos se puede interpretar que el queso es un derivado lácteo con alto valor nutritivo, debido a su perfil aminoacídico.

La proteína del queso posee alto valor biológico, según Walstra et al. (2006), define al queso como un sistema tridimensional tipo gel, formado básicamente por caseína integrada en un complejo caseinato fosfato cálcico, el cual por coagulación, engloba glóbulos de grasa, agua, lactosa, albúminas, globulinas, minerales, vitaminas y otras sustancias menores de la leche. El queso es un producto lácteo que contiene una elevada cantidad de grasas saturadas e insaturados, se diferencia de otras grasas alimenticias por su alto contenido de ácidos grasos saturados de cadenas cortas (Larsson, Virtamo y Wolk, 2012). La sustitución de grasa de la leche con aceite vegetal en la elaboración de queso cuenta con la ventaja que los aceites vegetales no tienen colesterol, es de bajo costo y es más estable a la variación estacional como presenta la grasa de la leche (LaBell et al., 1992). Existen diversos aceites vegetales que se pueden emplear para sustituir la grasa láctea, las semillas poseen aceites ricos en ácidos grasos saturados, entre estos tenemos las semillas de calabaza. Los contenidos de aceite en las semillas de calabaza fluctúan en muchas investigaciones desde un 30\% tal como menciona Bemis et al. (1967) hasta un 50\% como indica Murkovic et al. (2004). Por otro lado, además el contenido de ácidos esteárico y linoleico fluctúan de 3 a $8 \%$ y de 42 a $54 \%$, respectivamente. Respecto al contenido de ácido palmítico varían de 5,4 hasta $12 \%$ y de ácido oleico de 29\% a 43\% (Bemis et al., 1967; Jacks et al., 1972; Younis et al., 2000; Murkovic y Pfannhauser, 2000; Murkovic et al., 2004). El aceite de semillas de C. pepo L. presenta ácido linoleico de 43 a $56 \%$ y de ácido oleico de 24 a 38\%. (Bombardelli E, Morazzoni P.; 1997 y Younis Y, Ghirmay S.; 2000). Existen pocos estudios sobre el uso de aceites vegetales como sustituto de grasa de la leche en el proceso de fabricación de queso fresco; sin embargo, en el diseño la fabricación de quesos bajos en grasa, el papel fundamental de los aceites vegetales es de vital importancia ya que influye en sabor, textura de quesos bajos en grasa y especialmente en la aceptabilidad del consumidor. En tal sentido el objetivo es elaborar queso fresco con sustitución parcial de aceite de Cucúrbita ficifolia B. "Calabaza" y su evaluación fisicoquímica y sensorial.

\section{METODOLOGÏA}

Se utilizaron para la extracción de aceite de semilla de calabaza (Cucúrbita ficifolia B.) provenientes del distrito de Ahuac, provincia de Chupaca del departamento de Junín y la leche de vaca de la Estación Experimental del Mantaro de la Universidad Nacional del Centro del Perú. Se tomaron muestras representativas de $500 \mathrm{~g} \mathrm{se}-$ millas y 20 litros de leche fresca. Los reactivos utilizados fueron de grado analítico (marca Merck y Panreac).

El análisis fisicoquímico de la leche y queso fresco determinó el contenido de grasa, proteína y densidad. El contenido de lactosa y el contenido de sólidos totales por el método matemático Richmond; el pH se midió con un potenciómetro; la acidez por el método de titulación y; el contenido de cenizas por incineración de las muestras a $500-550{ }^{\circ} \mathrm{C}$ durante tres hora (NTP 202.001, 2003).

\section{Análisis del perfil de ácidos grados del aceite de semilla de Calabaza}

Las muestras fueron saponificadas y metiladas (de acuerdo con los procedimientos de la AOCS Ce 2-66). Los ésteres metílicos de los ácidos grasos (EMAG) fueron analizados en un cromatógrafo de gas (CG) (Shimadzu modelo 2000) equipado con un detector y un integrador de flama ionizante de acuerdo con el método descrito por Medina-Juárez et al. (2000).

\section{Obtención del queso fresco con incorporación de aceite de semilla de calabaza}

El desarrollo experimental de la elaboración del queso fresco fue en base al diagrama de flujo de la figura 1 .

\section{Diseño Experimental}

Se utilizó un diseño completamente al azar con seis tratamientos (leche estandarizada al 1 y 3\%; incorporación de aceites de semilla de calabaza 0,1;0,5 y 0,7\%). La unidad experimental fue la leche estandarizada. Las variables de respuesta fueron las características fisicoquímicas y sensoriales del queso fresco.

\section{Análisis estadístico de datos}

Los resultados fueron expresados como la media de seis tratamientos en forma independientes. Las diferencias significativas entre las medias, se calcularon mediante el análisis de varianza de una vía y una prueba de Tukey, con un nivel de significancia del 5\%. Se utilizó el programa estadístico SAS v8.0. 
Figura 1. Diagrama de flujo de elaboración de queso

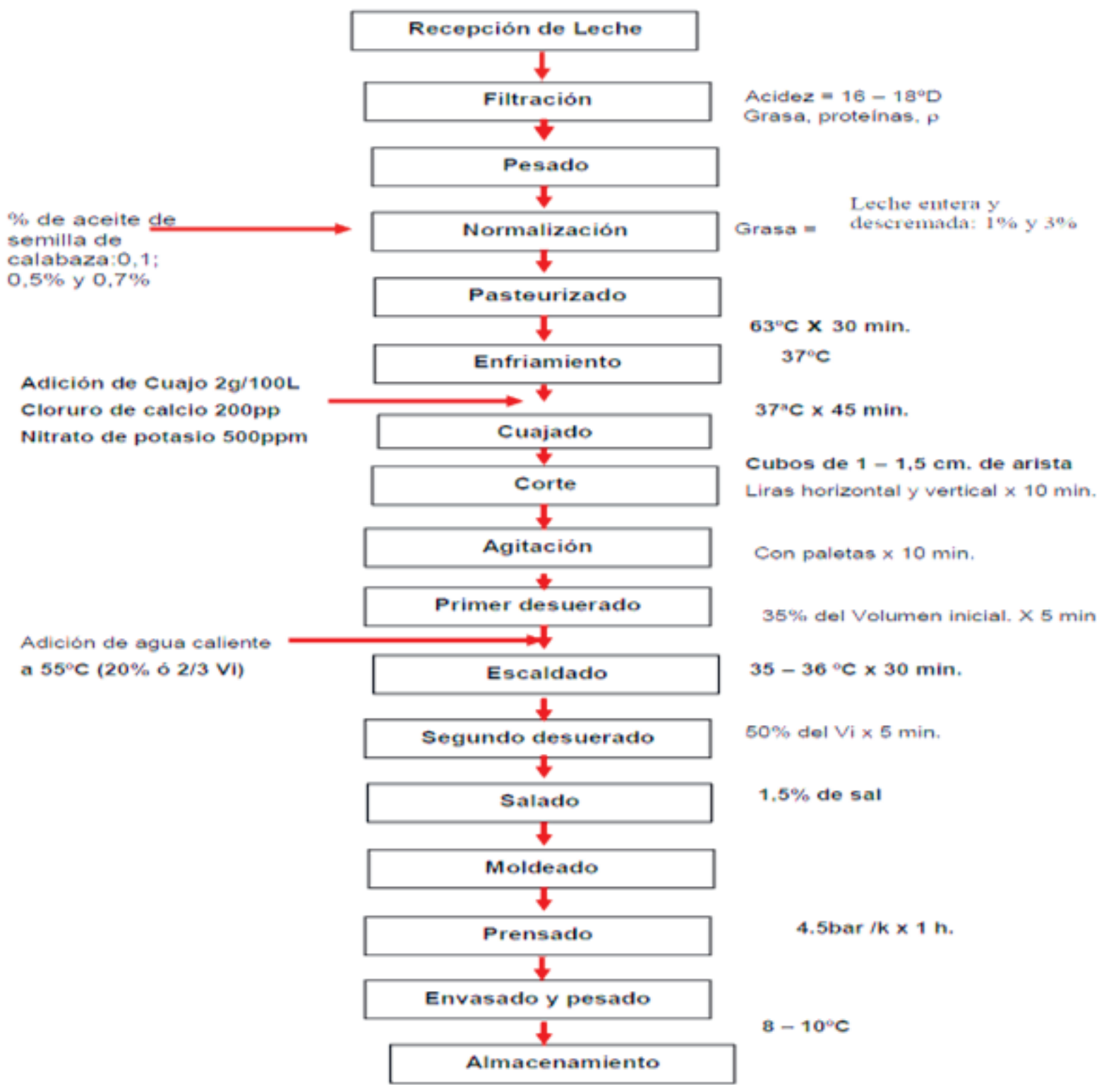

\section{RESULTADOS}

\section{Evaluación de materia prima}

En las tablas 01 y 02 , se presentan los resultados de la evaluación físico-química de la leche normalizada al 3\% $\pm 0,01$ y de sus principales componentes mayoritarios de la leche.

Tabla $\mathbf{N}^{\circ}$ 01. Análisis físico-químico de la leche al 3\%

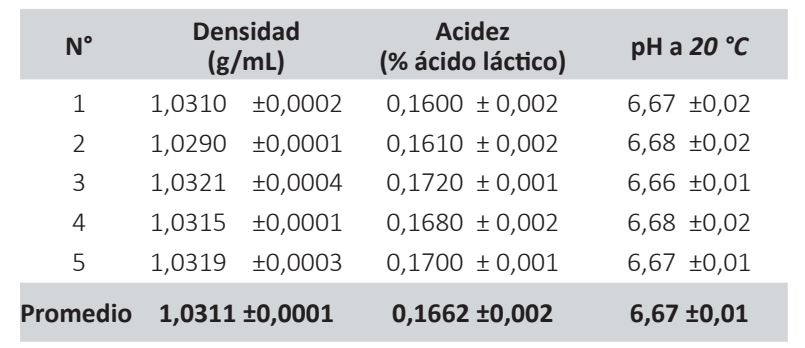

$n=$ Número de muestras

$m=$ Media muestral de los análisis para cada característica.

Tabla $\mathbf{N}^{\circ} \mathbf{0 2}$. Analisis químico proxima de la lecha al $3 \%$ de grasa $\mathbf{N}^{\circ}$ grasa \% proteínas \% lactosa \% Cenizas \% \begin{tabular}{l}
$\begin{array}{l}\text { sólidos } \\
\text { totales }\end{array}$ \\
\hline
\end{tabular}

$13,00 \pm 0,01 \quad 3,20 \pm 0,05 \quad 4,78 \pm 0,02 \quad 0,81 \pm 0,01 \quad 11,79 \pm 0,03$

$2 \quad 2,90 \pm 0,02 \quad 3,45 \pm 0,02 \quad 4,89 \pm 0,01 \quad 0,83 \pm 0,04 \quad 12,07 \pm 0,02$

$3 \quad 3,00 \pm 0,01 \quad 3,35 \pm 0,06 \quad 4,48 \pm 0,04 \quad 0,75 \pm 0,08 \quad 11,58 \pm 0,05$

$43,00 \pm 0,01 \quad 3,29 \pm 0,04 \quad 4,63 \pm 0,02 \quad 0,86 \pm 0,01 \quad 11,78 \pm 0,01$

$53,00 \pm 0,03 \quad 3,20 \pm 0,03 \quad 4,45 \pm 0,01 \quad 0,78 \pm 0,07 \quad 11,43 \quad 0,03$

$\mu \quad 2,98 \pm 0,01 \quad 3,29 \pm 0,04 \quad 4,64 \pm 0,02 \quad 0,80 \pm 0,04 \quad 11,73 \pm 0,03$

$n=$ Número de muestras

$m=$ Media muestral de los análisis para cada característica. 
En las tablas 03 y 04, se muestran los resultados de la evaluación fisicoquímica y químico proximal de la leche al $1 \%$ de grasa para la elaboración de queso fresco prensado. Los resultados se obtuvieron en base a la metodología de la NTP 202.001(2003) estandarizadas para leche y derivados lácteos.

Tabla $\mathbf{N}^{\circ}$ 03. Análisis físico-químico de leche descremada al $1 \%$ de grasa

\begin{tabular}{|c|c|c|c|c|}
\hline \multirow[b]{2}{*}{$\mathbf{N}^{\circ}$} & \multicolumn{4}{|c|}{ Características físico-químicas } \\
\hline & $\begin{array}{c}\text { Densidad }(\mathrm{g} / \mathrm{mL}) \\
\text { A } 20^{\circ} \mathrm{C}\end{array}$ & $\begin{array}{c}\text { Acidez } \\
\text { (\% Ácido Láctico) }\end{array}$ & $\mathrm{pH}$ a & $20^{\circ} \mathrm{C}$ \\
\hline 1 & $1,0350 \pm 0,0001$ & $0,1650 \pm 0,003$ & 6,68 & $\pm 0,01$ \\
\hline 2 & $1,0340 \pm 0,0003$ & $0,1640 \pm 0,003$ & 6,68 & $\pm 0,03$ \\
\hline 3 & $1,0360 \pm 0,0001$ & $0,1710 \pm 0,003$ & 6,68 & $\pm 0,04$ \\
\hline 4 & $1,0345 \pm 0,0005$ & $0,1780 \pm 0,002$ & 6,69 & $\pm 0,03$ \\
\hline 5 & $1,0356 \pm 0,0001$ & $0,1580 \pm 0,002$ & 6,69 & $\pm 0,02$ \\
\hline $\boldsymbol{\mu}$ & $1,0350 \pm 0,0003$ & $0,1671 \pm 0,003$ & 6,68 & $\pm 0,03$ \\
\hline
\end{tabular}

$m=$ Media muestral de los análisis para cada característica $n=$ Número de muestras

Tabla $\mathbf{N}^{\circ}$ 04. Análisis químico proximal de leche descremada al $1 \%$ de grasa

$\begin{array}{cccccc}\text { N } & \begin{array}{c}\text { Grasa } \\ \%\end{array} & \begin{array}{c}\text { Proteínas } \\ \%\end{array} & \begin{array}{c}\text { Lactosa } \\ \%\end{array} & \begin{array}{c}\text { Cenizas } \\ \%\end{array} & \begin{array}{c}\text { Sólidos } \\ \text { totales } \\ \%\end{array}\end{array}$

$1 \quad 1,10 \pm 0,01 \quad 3,16 \pm 0,04 \quad 4,35 \pm 0,01 \quad 0,68 \pm 0,02 \quad 9,29 \pm 0,02$

$21,30 \pm 0,01 \quad 3,20 \pm 0,04 \quad 4,32 \pm 0,03 \quad 0,60 \pm 0,03 \quad 9,42 \pm 0,04$

$31,10 \pm 0,02 \quad 3,25 \pm 0,06 \quad 4,37 \pm 0,02 \quad 0,79 \pm 0,01 \quad 9,51 \pm 0,03$

$4 \quad 1,10 \pm 0,01 \quad 3,27 \pm 0,04 \quad 4,50 \pm 0,01 \quad 0,77 \pm 0,02 \quad 9,64 \pm 0,02$

$5 \quad 1,00 \pm 0,02 \quad 3,15 \pm 0,04 \quad 4,49 \pm 0,04 \quad 0,75 \pm 0,02 \quad 9,39 \pm 0,01$

$\mu \quad 1,12 \pm 0,01 \quad 3,20 \pm 0.04 \quad 4,40 \pm 0,02 \quad 0,71 \pm 0,02 \quad 9,45 \pm 0,02$

$m=$ Media muestral de los análisis para cada característica $n=$ Número de muestra

Evaluación del Perfil de ácidos grasos del aceite de Cucurbita ficifolia B. "calabaza"

Tabla $\mathbf{N}^{\circ}$ 05. Comportamiento de ácidos grasos del aceite de semilla de cucurbita ficifolia B. "calabaza"

\begin{tabular}{ccc|} 
& \multicolumn{2}{c}{ Prensado en frío } \\
Ácidos grasos & Media\% & \pm desvstan \\
\hline Ácido palmítico & 8,898 & $\pm 0,253$ \\
Ácido esteárico & 4,161 & $\pm 0,157$ \\
Ácido oleico & 27,954 & $\pm 0,147$ \\
Ácido linoleico & 58,987 & $\pm 1,043$ \\
Ácido eicosanoico & - & -
\end{tabular}

Resultados de la evaluación físico-química del queso fresco con aceite de semilla de calabaza.

En la tabla 06, se muestran los resultados del compor- tamiento de las características fisicoquímicas del queso fresco prensado elaborado con leche estandarizada al 1 y $3 \%$ de grasa y sustitución de aceite de semilla de calabaza a niveles de 0,$1 ; 0,5$ y $0,7 \%$ respectivamente.

Tabla $\mathbf{N}^{\circ}$ 06. Acidez y $\mathrm{pH}$ del queso fresco prensado elaborado con incorporación de aceitede semilla de calabaza

\begin{tabular}{lcccccc}
\multicolumn{1}{c}{$\begin{array}{c}\text { Características } \\
\text { Físico-químicas }\end{array}$} & $\mathbf{0 , 1 \%}$ & $\mathbf{0 , 5 \%}$ & $\mathbf{0 , 7 \%}$ & $\mathbf{0 , 1 \%}$ & $\mathbf{0 , 5 \%}$ & $\mathbf{0 , 7 \%}$ \\
\hline $\mathrm{pH}$ a $20{ }^{\circ} \mathrm{C}$ & 6,10 & $\mathbf{6 , 2 0}$ & 5,90 & 5,95 & 5,60 & 5,80 \\
Acidez(\% ácido láctico) & 0,109 & 0,112 & 0,113 & 0,123 & 0,130 & $\mathbf{0 , 1 2 9}$
\end{tabular}

Resultados del análisis químico proximal de queso fresco elaborado con incorporación de aceite de semilla de calabaza

Los resultados, que se muestran en la tabla 07, corresponden al comportamiento de la evaluación químico proximal del queso fresco prensado a partir de leche estandarizada al 1 y $3 \%$ con incorporación de aceite de semilla de calabaza a niveles de $0,1 \% ; 0,5 \%$ y $0,7 \%$. Estas características que se muestran en dicha tabla son el resultado obtenido en base tres repeticiones. Además se muestran los resultados de la evaluación estadística de la interacción de los factores grasa de leche e incorporación de aceite de semilla de calabaza bajo los tres niveles de sustitución; estos resultados estadísticos se obtuvieron en base al software $S A S_{v} 8$.

Tabla 07. Evaluación químico proximal del queso fresco prensado en base a los tratamientos establecidos y su evaluación estadística

\begin{tabular}{|c|c|c|c|c|c|c|}
\hline \multirow{2}{*}{$\begin{array}{c}\text { Componentes } \\
\%\end{array}$} & \multicolumn{3}{|c|}{$1 \%$} & \multicolumn{3}{|c|}{$3 \%$} \\
\hline & $0,1 \%$ & $0,5 \%$ & $0,7 \%$ & $0,1 \%$ & $0,5 \%$ & $0,7 \%$ \\
\hline Grasa & $6,30^{c}$ & $6,70^{\mathrm{b}}$ & $6,80^{\mathrm{a}}$ & $14,30^{c}$ & $14,10^{b}$ & $14,20^{\mathrm{a}}$ \\
\hline $\begin{array}{l}\text { Proteínas } \\
\left(N^{*} 6,38\right)\end{array}$ & $18,23^{a}$ & $17,34^{\mathrm{b}}$ & $17,89^{c}$ & $20,45^{a}$ & $21,12^{b}$ & $19,34^{c}$ \\
\hline Lactosa & $6,89^{c}$ & $6,67^{b}$ & $6,82^{\mathrm{a}}$ & $7,12^{\mathrm{c}}$ & $7,28^{b}$ & $7,37^{\mathrm{a}}$ \\
\hline Cenizas & $3,68^{\mathrm{c}}$ & $3,67^{a}$ & $3,71^{\mathrm{b}}$ & $3,66^{c}$ & $3,87^{\mathrm{a}}$ & $3,76^{b}$ \\
\hline Sólidos totales & $35,10^{\mathrm{b}}$ & $34,38^{\mathrm{a}}$ & $35,22^{c}$ & $45,53^{b}$ & $46,37^{a}$ & $44,67^{c}$ \\
\hline Humedad & $64,90^{b}$ & $65,62^{c}$ & $64,78^{\mathrm{a}}$ & $54,47^{b}$ & $53,63^{c}$ & $55,33^{\mathrm{a}}$ \\
\hline
\end{tabular}

Resultados de la evaluación de rendimiento de queso fresco prensado elaborado con incorporación de aceite de semilla de calabaza

La evaluación del rendimiento quesero en base a los tratamientos desarrollados se muestra en la tabla 10, donde estos rendimientos corresponden a tres lotes elaborados por cada tratamiento, obteniéndose un promedio de cada lote elaborado.

Tabla $\mathbf{N}^{\circ}$ 08. Rendimiento quesero en base a los tratamientos propuestos 


\begin{tabular}{ccccccc}
$\begin{array}{c}\text { Rendimiento } \\
\text { (\%) } \\
\text { Lote }\end{array}$ & $\mathbf{0 , 1 \%}$ & $\mathbf{0 , 5 \%}$ & $\mathbf{0 , 7 \%}$ & $\mathbf{0 , 1 \%}$ & $\mathbf{0 , 5 \%}$ & $\mathbf{0 , 7 \%}$ \\
\hline 1 & 12,89 & 13,24 & 13,76 & 14,10 & 13,85 & 14,75 \\
2 & 12,38 & 12,68 & 12,72 & 13,89 & 14,26 & 14,28 \\
3 & 12,50 & 12,58 & 12,37 & 13,78 & 14,15 & 14,60 \\
\hline Promedio & $\mathbf{1 2 , 5 9}$ & $\mathbf{1 2 , 8 3}$ & $\mathbf{1 2 , 9 5}$ & $\mathbf{1 3 , 9 2}$ & $\mathbf{1 4 , 0 8}$ & $\mathbf{1 4 , 5 4}$ \\
\hline
\end{tabular}

Resultados de la evaluación sensorial del queso fresco prensado elaborado con incorporación de aceite de semilla de calabaza

Los resultados de la evaluación sensorial en su aceptabilidad se presentan en la tabla 11 , las cuales se obtuvieron utilizando 30 panelistas entrenados para evaluar los atributos señoriales del queso fresco en base a una escala hedónica de 1 a 9.

La valoración final fue en función al puntaje obtenido por cada atributo y comparado con un queso elaborado sin la incorporación de aceite de semilla de calabaza como testigo.

Tabla $\mathbf{N}^{\circ}$ 09. Valoración de la evaluación sensorial del queso fresco prensado con incorporación de aceite de semilla de calabaza

\begin{tabular}{rrcccc}
\multicolumn{2}{c}{ Tratamientos } & Color & Olor & Sabor & Textura \\
\hline & T0.1 & 138 & 113 & 136 & 126 \\
& T0.5 & 158 & 139 & 148 & 144 \\
& T0.7 & 142 & 127 & 109 & 110 \\
& Testigo & 162 & 144 & 154 & 150 \\
& T0.1 & 116 & 131 & 102 & 116 \\
& T0.5 & 110 & 123 & 110 & 121 \\
& T0.7 & 108 & 124 & 102 & 120 \\
& Testigo & 162 & 144 & 154 & 150
\end{tabular}

\section{DISCUSIÓN}

La leche estandarizada presenta una densidad promedio para las cinco muestras un valor de $1.0311 \pm 0,0001$, una acidez de $0,1662 \% \pm 0,002$ (expresado en ácido láctico), con un $p H$ de 6,67 $\pm 0,01$. Estos resultados obtenidos están dentro de los requisitos que deben cumplir la leche destinada a la producción de queso fresco tal como como indica Walstra et al. (2006), que la leche debe presentar un $p H$ de 6,5 a 6,75, una acidez de 13,95 - 16,2 ${ }^{\circ} \mathrm{D}(0,1395$ a $0,162 \%$ expresado en ácido láctico) y un contenido de solidos totales de $11 \%$. En las tablas $01 \mathrm{y}$ 02 , se presenta un contenido promedio de grasa de $3 \%$ y un porcentaje de sólidos totales de 11,73\%.

En las tablas 03 y 04, se pueden observar los resultados de la evaluación de la leche estandarizada al 1\% de grasa, en donde presenta una densidad promedio para las ocho muestras un valor de 1,035 $\pm 0,0003$, un $\mathrm{pH}$ de 6,68 \pm
0,03 y una acidez de 0,1671\% $\pm 0,003$ (expresado en ácido láctico); contenido de lactosa de 4,40\% $\pm 0,02$ y un 9,45\% $\pm 0,02$ de sólidos totales; como se observa hay una variación substancial de los macro componentes de la leche por efecto del descremado; si se compara con la leche estandarizada al 3\%, hay un ligero incremento en la densidad y en el $p H$, esto se debe fundamentalmente al efecto del procesamiento en la operación del descremado. En la Tabla 05, el aceite se semilla de calabaza presenta un alto contenido de ácido linoleico con un promedio de 58,987\%; estos valores se asemejan a lo reportado por Petkove y Antova (2014); lo que incrementa la importancia de utilizar aceite de semilla de calabaza para elaborar queso fresco con valor nutritivo en relación a la calidad de grasa contenida. La Tabla 9, demuestra que la variación del contenido de proteínas, lactosa, cenizas, humedad y sólidos totales están dentro de los valores normativos vigentes en la NTP (2000); respecto a proteínas fluctúan según los tratamiento experimental de un valor mínimo de $17,34 \%$ hasta un valor máximo de $421,12 \%$; en lo que corresponde a grasa se obtiene un valor mínimo de $6,30 \%$ hasta valor máximo de $14,30 \%$; lactosa del mismo modo de 6,67\% hasta 7,37\%; humedad de 55,33\% hasta un valor máximo de 65,62\% respectivamente. Sin embargo, Madrid (1996), presenta un clasificación de quesos: quesos cremosos, semicremosos o descremados, cosidos o, simplemente de leche quesos elaborados de leche pasteurizada; queso de leche descremada: tienen un mínimo de $30 \%$ de proteína y $50 \%$ de humedad, como máximo y queso de leche parcialmente descremada: presentan un mínimo de $18 \%$ de grasa y $30 \%$ de proteínas y, como máximo, $48 \%$ de humedad respectivamente.

Según estos resultados, los rendimientos están en función directa a la concentración de los sólidos totales de la leche; tal como se puede apreciar en la Tabla 08, donde a mayor concentración de solidos totales, mayor rendimiento quesero; sin embargo, es necesario indicar que los niveles $(0,1$; 0,5 y $0,7 \%$ ) de incorporación de aceite de semilla de calabaza en la elaboración de queso fresco prensado no influye directamente en el rendimiento quesero. Por otro lado, Revilla (1982) menciona los diferentes tipos de queso elaborados tradicionalmente, su rendimiento está influenciado por la concentración de materia seca y el contenido de humedad en el producto final que varían de 12 a $14 \%$.

En la Tabla 09, se muestran los resultados de la valoración sensorial de los atributos del queso fresco en base a una escala hedónica, de 1 a 9 para 30 panelistas entrenados; el tratamiento que sobresale, según los puntajes que se obtuvieron son: para el color, olor, sabor y textura. El tratamiento (T0.5), donde el queso fresco se elaboró con leche al $1 \%$ de grasa y con $0,5 \%$ de incorporación de aceite de semilla de calabaza, y al comprar con el testigo, el queso fresco prensado con leche al $1 \%$ y $3 \%$ de grasa sin incorporación de aceite presentan relativamente valores cercanos al puntaje obtenido por la muestra testigo. Por lo tanto, existe una preferencia en los cuatro 
atri- butos evaluados en comparación a la muestra testigo, al utilizar $0,5 \%$ de aceite de semilla y con $1 \%$ de grasa de leche, bajo estas concentraciones de sustitución de la grasa de leche por el aceite de calabaza se obtiene queso fresco prensado con los mismos atributos tal como presentan el queso fresco prensado comercial; con la ventaja nutricional que el aceite de semilla de calabaza aporta ácidos grasos insaturados, y el queso fresco prensado, de bajo contenido de grasa, beneficia a una sector de la población, considerando que nutricionalmente estos ácidos grasos cumplen un función más importante comparado a la grasa de origen animal, tal como indican otros estudios re- lacionados (Alais, 2000). Losada y Serrano (2002) mencionan que los atributos o propiedades de los quesos están influenciados por diversos factores, como son: materia prima, tipo de coagulación, tipo de maduración, etc.); y además, depende del proceso tecnológico que se utilizan en la elaboración de los quesos, por lo que existen muchas variedades de queso tradicionales o con denominación de origen.

\section{CONCLUSIONES}

- Los quesos frescos elaborados a partir de leche estandarizada con $1 \%$ y $3 \%$ de grasa respectivamente, y con niveles de incorporación de aceite de semilla de calabaza de $0,1 \%, 0,5 \%$ y $0,7 \%$ presentan diferencias significativas en las características fisicoquímicas entre los tratamientos.

- La evaluación sensorial indica que al utilizar leche estandarizada al 1\% de grasa con un nivel de incorporación de aceite de semilla de calabaza; de $0,5 \%$, el queso fresco presenta mayor aceptabilidad mejorando su valor nutritivo en relación a la calidad de la grasa, gracias al perfil de ácidos grasos que presenta el aceite vegetal utilizado.

\section{REFERENCIAS BIBLIOGRÁFICAS}

Alais CH. 2000; Ciencia de la leche; edit. Reverte, 2da. Edic. México

Bemis, W.; Berry, W.; Kennedy, M.; Woods, D.; Morán, M. \& Deutschman Jr. A. 1967. Oil composition of Cucurbita. Journal of the American Oil Chemists' Society 44: 429-430.

Bombardelli, E. y Morazzoni, P. Cucurbita pepo L. Fitoterapia. 1997; 68(4):291-301. 5.

El-Adawy, T. \& Taha K. 2001. Characteristics and composition of different seed oils and flours. Food Chemistry 74: 47 -54.

Garzón, S. Obtención de metabolitos secundarios del extracto polar de la semilla de Cucurbita máxima y Cucurbita fisifolia [Tesis de Grado]. Cali, Ecuador: Universidad delValle; 1996.

Gómez, J. y Navas, S. “Recolección y caracterización morfológica molecular de accesiones de zambo (Cucúrbita ficifolia) en el Cantón Cotacachi [tesis]. Ibarra, Ecuador: Pontificia Universidad Católica del Ecuador; 2007

Jacks, T.; Hensarling, T. \& Yatsu, L. 1972. Cucurbit seeds: I. Characterizations and uses of oils and proteins. A Review. Economic Botany 26: 135-141.

LaBell. F.; Eilers, J. \& Duxbury, D. (1992) Current dairy research highlights low-fat cheese, food process. 53:41-47.

Larsson SC, Virtamo J, Wolk A. Dairy consumption and risk of stroke in Swedish women and men. Stroke. 2012; 43(7):1775-80.

Lee D.; Noh B.; Bae S. \& Kim K. 1998. Characterization of fatty acids composition in vegetable oils by gas chromatography and chemometrics. Analytica Chimica Acta 358: 163-175.

Liepa, G. \& Han-Markey, T. \& Sutton, M. 2000. Nutritional and health aspects of dietary lipids. Ch. 4. In: Introduction to fats and oils technology. O'Brien R. D.; Farr W. E.; Wan P. J. (Eds.). Illinois, USA: AOCS Press. pp. 63-81.

Medina, L.; Gámez, N.; Ortega, J.; Noriega, J. \& Angulo, O. 2000. Trans fatty acid composition and tocopherol content in vegetable oils produced in Mexico. Journal of the American Oil Chemists' Society 77: 721-724.

Murkovic, M.; Piironen, V.; Lampi, A.; Kraushofer, T. \& Sontag, G. 2004. Changes in chemical composition of pumpkin seeds during the roasting process for production of pumpkin seed oil. Part 1: non-volatile compounds. Food Chemistry 84: 359-365.

Murkovic, M. \& Pfannhauser, W. 2000. Stability of pumpkin seed oil. European Journal of Lipid Science andTechnology 102: 607-611.

Murkovic, M.; Piironen, V.; Lampi, A.; Kraushofer, T. \& Sontag, G. 2004. Changes in chemical composition of pumpkin seeds during the roasting process for production of pumpkin seed oil. Part 1: non-volatile compounds. Food Chemistry 84: 359-365.

Naz, S.; Siddiqi, R.; Sheik, H. \& Sayeed S. 2005. Deterioration of olive, corn and soybean oils due to air, light, heat and deep-frying. Food Research International 38: 127-134.

Norma Técnica Peruana NTP 202.001 2003 Leche y productos lacteos. Leche cruda. Requisitos.

Pérez, F.; Ruano, J.; Pérez, P.; López, F. y López, J. The influence of olive oil on human health: not a question of fat alone. Mol Nutr Food Res. 2007; 51:1199—208.

Petkove y Antova. 2014. Composición de ácidos grasos por cromatografía de gases de la Cucúrbita máxima B “calabaza”. [Tesis de grado]. Ecuador.

SAS Institute. Wan P. 2000. Properties of fats and oils. Ch. 2. In: Introduction to Fats and Oils Technology. O'Brien, R.; Farr, W.; Walstra, P.; Wouters, J. \& Geurts, T. 2006. Dairy Science and Technology. CRC Press. Nueva York, EE.UU. 140-155 pp.

White, P. 2000. Flavor quality of Fats and Oils. Ch. 18. In: Introduction to fats and oils technology. O'Brien, R.; Farr, W. \& Wan P. (Eds.). Illinois, USA: AOCS Press. pp. 341-370.

Younis, Y.; Ghirmay, S. \& Al-Shihry, S. 2000. African Cucurbita pepo L.: properties of seed and variability in fatty acid composition of seed oil. Phytochemistry 54: 71-75.

Younis, Y.; Ghirmay, S. \& Al-Shihry, S. African Cucurbita pepo L. properties of seed and variability in fatty acid composition of seed oil. Phytochem. 2000; 54(1):71-5. 